\title{
The use of del Nido cardioplegia in adult cardiac surgery: A prospective randomized trial
}

\author{
Niv Ad, MD, ${ }^{\mathrm{a}, \mathrm{b}, \mathrm{c}}$ Sari D. Holmes, PhD, ${ }^{\mathrm{d}}$ Paul S. Massimiano, MD, ${ }^{\mathrm{b}}$ Anthony J. Rongione, MD, \\ Lisa M. Fornaresio, $\mathrm{PhD},{ }^{\mathrm{d}}$ and David Fitzgerald, $\mathrm{MPH}, \mathrm{CCP}^{\mathrm{e}}$
}

\section{ABSTRACT}

Objectives: The del Nido cardioplegia solution has been used extensively in congenital heart surgery for more than 20 years and more recently for adults. This randomized controlled trial examined whether expanding this technique to adult cardiac surgery confers benefits in surgical workflow and clinical outcome compared with blood-based cardioplegia.

Methods: Adult first-time coronary artery bypass grafting (CABG), valve, or $\mathrm{CABG} /$ valve surgery patients requiring cardiopulmonary bypass (CPB) were randomized to del Nido cardioplegia $(n=48)$ or whole blood cardioplegia $(n=41)$. Primary outcomes assessed myocardial preservation. Troponin I was measured at baseline, 2 hours after CPB termination, 12 and 24 hours after cardiovascular intensive care unit admission. Alpha was set at $P<.001$.

Results: Preoperative characteristics were similar between groups, including age, Society of Thoracic Surgeons risk score, CABG, and valve procedures. There was no significant difference on CPB time (97 vs 103 minutes; $P=.288$ ) or crossclamp time (70 vs 83 minutes; $P=.018$ ). The del Nido group showed higher return to spontaneous rhythm $(97.7 \%$ vs $81.6 \% ; P=.023)$ and fewer patients required inotropic support $(65.1 \%$ vs $84.2 \% ; P=.050)$, but did not reach statistical significance. Incidence of Society of Thoracic Surgeons-defined morbidity was low, with no strokes, myocardial infarctions, renal failure, or operative deaths. For del Nido group patients, troponin levels did not increase as much as for control patients $(P=.040)$, but statistical significance was not reached.

Conclusions: Evidence from this study suggests del Nido cardioplegia use in routine adult cases may be safe, result in comparable clinical outcomes, and streamline surgical workflow. The trend for troponin should be investigated further because it may suggest superior myocardial protection with the del Nido solution. (J Thorac Cardiovasc Surg 2018;155:1011-8)

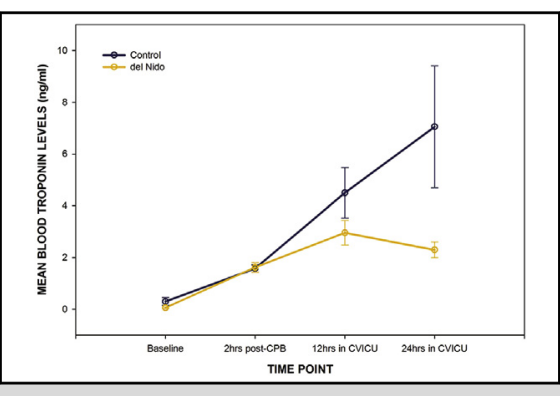

Mean troponin I levels by treatment group from presurgery to 24 hours after cardiovascular intensive care unit (CVICU) admission.

\section{Central Message}

Evidence from this initial randomized controlled trial suggests use of del Nido cardioplegia in routine adult cases may be safe, have comparable clinical outcomes, and streamline surgical workflow.

\section{Perspective}

The del Nido cardioplegia solution has been used extensively in congenital heart surgery for more than 20 years. Recent literature has suggested that del Nido solution may be administered safely in adult surgical procedures. Results of this randomized trial support previous studies on safety of del Nido solution and suggest comparable results for myocardial injury and potential benefits in surgical workflow.

See Editorial Commentary page 1019.

See Editorial page 1009.

\footnotetext{
From the ${ }^{a}$ Department of Cardiovascular and Thoracic Surgery, West Virginia University, Morgantown, WVa; ${ }^{\mathrm{b}}$ Adventist HealthCare, Takoma Park, Md; ${ }^{\mathrm{c} I n o v a}$ Fairfax Hospital, Falls Church, Va; ${ }^{\mathrm{d}}$ Heart and Vascular Institute, West Virginia University Medicine, Morgantown, WVa; and ${ }^{\mathrm{e}}$ The Medical University of South Carolina, Charleston, SC.

Read at the 97th Annual Meeting of The American Association for Thoracic Surgery, Boston, Massachusetts, April 29-May 3, 2017.

Received for publication May 17, 2017; revisions received Sept 8, 2017; accepted for publication Sept 22, 2017; available ahead of print Dec 12, 2017.

Address for reprints: Niv Ad, MD, Department of Cardiovascular and Thoracic Surgery, West Virginia University, 1 Medical Center Dr, Morgantown, WV 26506 (E-mail: nivadmd14@gmail.com).

$0022-5223 / \$ 36.00$

Copyright (C) 2017 by The American Association for Thoracic Surgery

https://doi.org/10.1016/j.jtcvs.2017.09.146
}

Optimal myocardial protection during cardiac surgery is a key component of a successful procedure. ${ }^{1}$ Since the 1950s, research and strategies have been developed to continuously improve myocardial protection and prevent

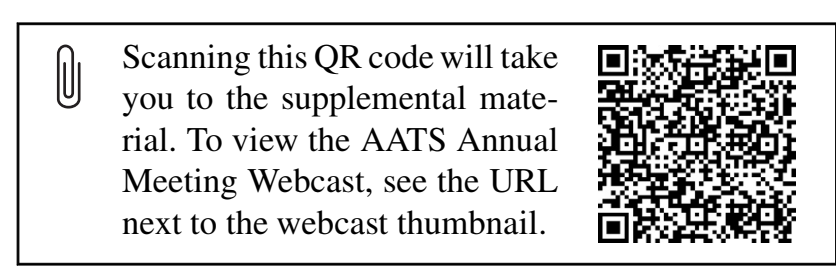




\section{Abbreviations and Acronyms \\ $\mathrm{CABG}=$ coronary artery bypass grafting \\ $\mathrm{CPB}=$ cardiopulmonary bypass \\ CVICU $=$ cardiovascular intensive care unit \\ STS = Society of Thoracic Surgeons \\ TEE $=$ transesophageal echocardiography}

further ischemic injury. ${ }^{2-4}$ The use of hypothermic and hyperkalemic cardioplegia solutions evolved to become the clinical standard in many cardiac surgery programs across the world. ${ }^{5}$ Despite the multitude of reported cardioplegia systems, there is a lack of clear consensus on composition, route, and technique. ${ }^{1,5,6}$

A novel formula for cardioplegia was introduced in congenital heart surgery in 1995 . $^{7}$ This formula was patented as a single dose and modified depolarizing solution. Now commonly referred to as del Nido solution, this blood and crystalloid mixed formula has been associated with a longer duration of safe myocardial ischemic arrest. ${ }^{8,9}$ This solution has been purported to preserve intracellular high-energy phosphates and intracellular $\mathrm{pH}$ and reduce calcium ion influx during and after ischemic arrest and led to its increased use across pediatric congenital cardiac surgery programs. ${ }^{10,11}$

Multiple observational studies have suggested that the del Nido solution is associated with safety and efficacy in adult surgical procedures. ${ }^{12-18}$ Despite these encouraging findings, adaptation of the protocol in treating adult ischemic myocardium has not been addressed by prospective randomized trials. ${ }^{19}$ The purpose of this prospective randomized controlled trial was to compare the del Nido cardioplegia protocol with an existing whole blood-based cardioplegia strategy in routine coronary artery bypass grafting $(\mathrm{CABG})$, valve, and $\mathrm{CABG} /$ valve surgery procedures.

\section{METHODS}

After obtaining institutional review board approval (study No.14-1653), adult patients presenting for first-time CABG or heart valve surgery requiring cardiopulmonary bypass (CPB) between February 2015 and April 2016 were eligible to participate (study No. NCT02442050). Inclusion criteria included adult patients aged 19 to 79 years; patients able to receive and provide informed consent; stable patients with surgical procedures requiring $\mathrm{CPB}$ and myocardial arrest; and isolated CABG surgery, isolated single-valve surgery, or concomitant $\mathrm{CABG}$ and single-valve surgery. Exclusion criteria included previous cardiac surgery, patients with preoperative inotropic pharmacologic support, patients receiving preoperative mechanical circulatory support, patients with an implanted pacemaker or implantable cardioverter-defibrillator, and patients undergoing cardiac surgical procedures outside of the inclusion criteria categories.

Patients completed written informed consent and were randomized to receive del Nido cardioplegia solution or whole blood cardioplegia. There were 2 arms for randomization in this trial to ensure equitable representation of surgical procedures in each treatment group: all eligible patients undergoing any heart valve surgical procedure with or without CABG surgery, and all eligible patients undergoing CABG surgery. Within each arm, patients were randomized to the intervention group (del Nido solution) or the control group (whole blood cardioplegia) using random number generation and blocked randomization with groups of 20 . The randomization order was created before enrollment began and was then transferred to sealed consecutively numbered envelopes by a member of the study team who was not involved in patient enrollment. Of 99 patients who provided written informed consent and were enrolled in the study, 90 were randomized to a treatment group and 9 patients withdrew or had surgery canceled. Randomization occurred the day before the originally scheduled surgery date. One patient randomized to the control group was excluded because surgery was canceled. Therefore, 89 patients were included in these analyses (Figure 1) with 48 in the del Nido group and 41 in the control group.

Primary outcome measures assessed myocardial preservation by return to spontaneous rhythm; defibrillation requirement; inotropes; and troponin levels at 4 time points: baseline (at anesthesia induction), 2 hours after termination of CPB, 12 hours after admission to a cardiovascular intensive care unit (CVICU), and 24 hours after admission to a CVICU. Secondary outcomes assessed safety and workflow, including CPB and cross-clamp time, cardioplegia redosing, blood transfusion, and electrocardiogram changes 1 day after surgery. Postoperative clinical outcomes were defined by the Society of Thoracic Surgeons (STS) Adult Cardiac Surgical Database. Calculated ejection fraction was assessed using transesophageal echocardiography (TEE) before surgery and at the termination of CPB.

\section{CPB and Cardioplegia Delivery}

For these patients, CPB was conducted with the Terumo Advanced System I Extracorporeal Perfusion System (Terumo Cardiovascular Group, Ann Arbor, Mich). All CPB circuits consisted of a Terumo RX series oxygenator and hardshell reservoir, an AF-125 arterial filter, and Xcoating surface coating. Patients with a body surface area $<1.9 \mathrm{~m}^{2}$ received a Terumo RX-15 oxygenator and 3/8-in arterial and venous tubing, whereas patients with a body surface area $\geq 1.9 \mathrm{~m}^{2}$ were supported with a Terumo RX-25 oxygenator and 1/2-in venous tubing. Heparin dosing and anticoagulation was calculated using the Medtronic Hemostasis Management System (Medtronic, Minneapolis, Minn). Acute normovolemic hemodilution and retrograde autologous prime were performed on all patients deemed hemodynamically stable to donate and able to maintain a CPB hematocrit of at least $21 \%$. Target hypothermic systemic temperatures on CPB support were maintained between $32^{\circ} \mathrm{C}$ and $35^{\circ} \mathrm{C}$. The use of cardiotomy pump suction was limited to intracardiac blood collected during heart valve procedures. The Fresenius Continuous Autotransfusion System (CATS ${ }^{\text {plus }}$; Fresinius KABI AG, Bad Homburg, Germany) was used to process surgical shed blood and residual $\mathrm{CPB}$ circuit volume after bypass was complete.

The del Nido and whole-blood cardioplegia formulas were delivered through the Quest Medical Myocardial Protection System II (Quest Medical Inc, Allen, Tex) (Table 1). Patients enrolled in the intervention group received $1.0 \mathrm{~L}$ del Nido cardioplegia after the aortic cross-clamp was applied to the ascending aorta (Central Admixture Pharmacy Services Inc, Bethlehem, Pa). An additional $500 \mathrm{~mL}$ was administered if left ventricular hypertrophy was present per the discretion of the attending surgeon. When a retrograde dose of cardioplegia through a coronary sinus catheter was requested by the surgeon, the calculated induction dose was equally split between antegrade and retrograde routes. Although not required for the study protocol, all patients received both antegrade and retrograde administration per standard of care. Subsequent doses of del Nido were not indicated unless the ischemic duration exceeded 90 minutes or there was spontaneous return of electrical activity during the aortic cross-clamp period. If a subsequent dose of del Nido was required, an additional $500 \mathrm{~mL}$ was administered. Delivery of del Nido solution was administered in a 1:4 ratio of blood to crystalloid at a temperature of $6^{\circ} \mathrm{C}$ to $10^{\circ} \mathrm{C}$. Topical cooling was applied in all cases.

Patients enrolled in the control group received whole blood cardioplegia according to the institutional adult cardioplegia protocol. No additional 


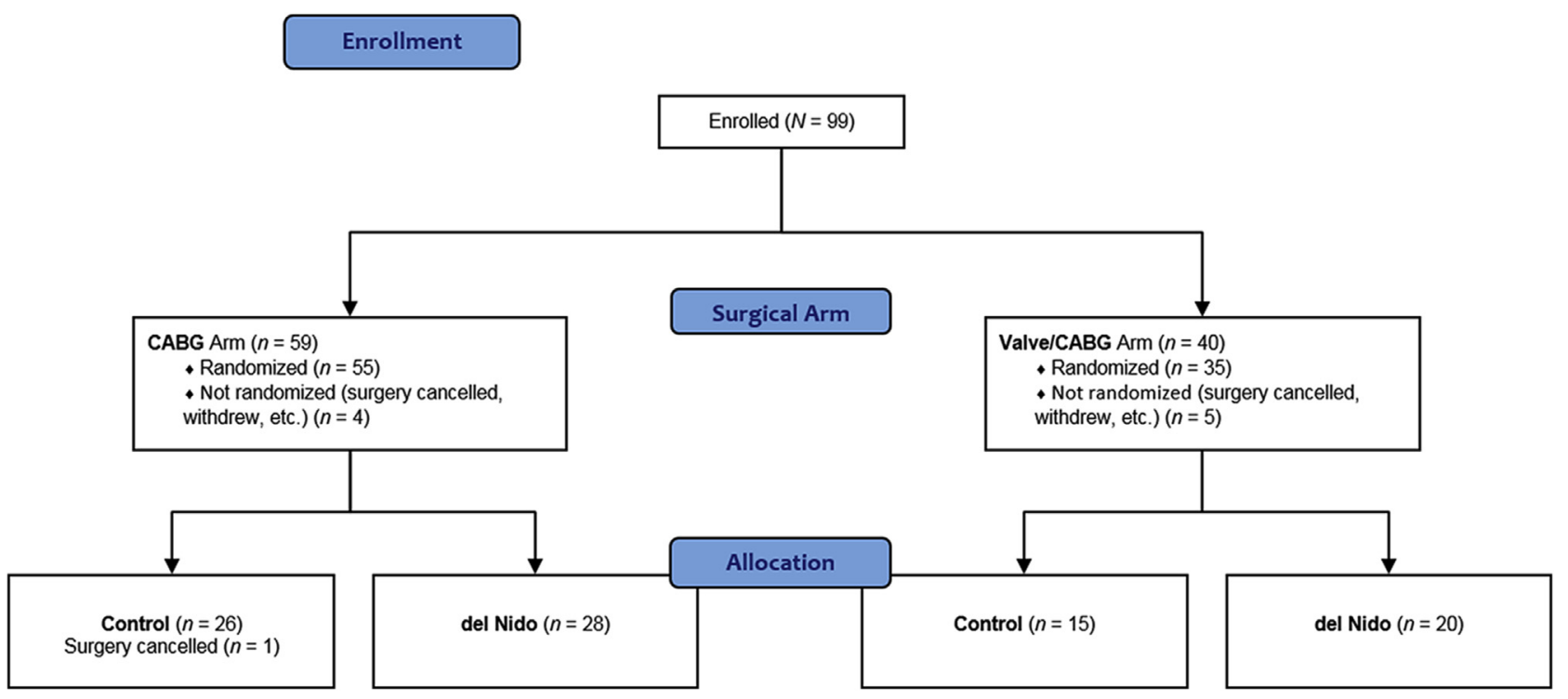

FIGURE 1. Enrollment flow diagram. $C A B G$, Coronary artery bypass grafting.

crystalloid solution was added to the mircoplegia formula; rather, potassium chloride and magnesium sulfate were titrated into the blood delivery. The dose and frequency of delivery was administered according to surgical protocol. The induction dose of whole blood cardioplegia ranged from 1.0 to $2.0 \mathrm{~L}$ of solution, with subsequent doses of cardioplegia approximately every 20 minutes. Cardioplegia doses were administered at a temperature of $8^{\circ} \mathrm{C}$ to $11^{\circ} \mathrm{C}$. Before removal of the aortic cross-clamp, a dose of amino acid-enriched warm cardioplegia solution, monosodium aspartate, and glutamate was provided, as well as a systemic bolus of $1 \%$ lidocaine sulfate of $2 \mathrm{mg} / \mathrm{kg}$ (maximum dose of $200 \mathrm{mg}$ ). TEE was used to perform pre- and post-CPB left ventricular ejection fraction calculations. The need for pharmacologic post-CPB inotropic support was at the discretion of the surgeon and anesthesiologist.

TABLE 1. Cardioplegia formulas and strategy by treatment group

\begin{tabular}{|c|c|c|}
\hline Formula & $\begin{array}{c}\text { del Nido } \\
\text { (treatment) }\end{array}$ & $\begin{array}{c}\text { Whole blood } \\
\text { (control) }\end{array}$ \\
\hline Carrier solution & $\begin{array}{r}\text { Plasmalyte-A } \\
(1000 \mathrm{~mL})\end{array}$ & Whole blood \\
\hline Blood:crystalloid & $1: 4$ & $\begin{array}{l}\text { Whole blood with } \\
\text { titration }\end{array}$ \\
\hline Potassium chloride & $26 \mathrm{mEq}$ & $\begin{array}{l}25 \mathrm{mEq} / \mathrm{L} \text { induction } \\
6-10 \mathrm{mEq} / \mathrm{L} \\
\quad \text { maintenance }\end{array}$ \\
\hline Mannitol (20\%) & $3.26 \mathrm{~g}$ & 0 \\
\hline Lidocaine (1\%) & $130 \mathrm{mg}$ & 0 \\
\hline Magnesium sulfate $(50 \%)$ & $2 \mathrm{~g}$ & $\begin{array}{l}1 \mathrm{mg} / \mathrm{mL} \text { induction } \\
0.5 \mathrm{mg} / \mathrm{mL} \\
\text { maintenance }\end{array}$ \\
\hline Sodium bicarbonate $(8.4 \%)$ & $13 \mathrm{mEq}$ & 0 \\
\hline $\begin{array}{l}\text { Monosodium aspartate/ } \\
\text { Glutamate ( } 0.92 \text { molar })\end{array}$ & 0 & $50 \mathrm{~mL} / \mathrm{L}$ \\
\hline
\end{tabular}

\section{Troponin Assessment}

Blood was collected from patients to perform serum troponin assays at 4 scheduled time points: at anesthesia induction, 2 hours after the discontinuation of $\mathrm{CPB}, 12$ hours after admission into the CVICU, and 24 hours after admission to the CVICU. The Architect Stat Troponin-I assay (Abbott Laboratories, Abbott Park, Ill) is a 2-step immunoassay to determine the presence of cardiac troponin in human plasma using chemiluminescent microparticle immunoassay technology.

\section{Statistical Analyses}

All analyses were conducted using IBM SPSS Statistics version 24.0 (IBM Corp, Armonk, NY). Noninferiority analyses were originally planned for the primary study outcomes assuming there would be no differences between the 2 treatments. Power analyses indicated a sample size of 500 patients $(\mathrm{n}=250$ per group) would be sufficient to support noninferiority of del Nido with the originally planned analyses using a 5\% acceptable difference margin (noninferiority limit) based on previous data from Ovrum and colleagues. ${ }^{1}$ However, when interim analyses that were built into the protocol for data safety monitoring were conducted to assess the safety of the treatment arms, meaningful differences beyond noninferiority were found. Analyses were therefore shifted to a superiority methodology to assess the differences further and the study was stopped at this point. Based on results of the interim analyses presented here, there was no justification to continue the study for noninferiority objectives given the differences in outcomes. Subsequently, a post hoc alpha spending function using the Lan-DeMets method was applied to evaluate how the alpha level was affected by stopping the study at this point. ${ }^{20,21}$ An alpha level of $P<.001$ was determined to be required at this point in the study for statistical significance to be achieved. Therefore, only a $P$ value of $<.001$ two-tailed was considered statistically significant. However, given the preliminary nature of this study, results that suggest potential for statistical significance at a traditional alpha level of $P<.05$ in a larger study are reported.

Continuous data are presented as mean \pm standard deviation or median (interquartile range) and categorical data are presented as frequency $(\%)$. Analyses comparing patient characteristics and outcomes by treatment group included independent-sample $t$ tests or the Mann-Whitney $U$ test for continuous variables and $\chi^{2}$ or Fisher exact tests for categorical variables. A repeated measures analysis of variance testing the main effect 
of time, the main effect of treatment, and the time $\times$ treatment interaction was conducted to examine the change in troponin levels over the 4 time points by treatment group. Complete data on all 4 time points was required for this analysis and listwise deletion was employed for patients with missing troponin data (control $\mathrm{n}=2$ and del Nido $\mathrm{n}=3$ ). Linear regression evaluated the effect of treatment group on ejection fraction at the termination of CPB after adjustment for preoperative ejection fraction.

\section{RESULTS}

The treatment groups were similar on all preoperative clinical characteristics, including age (65.3 vs 65.1 years), STS risk score (1.4\% vs $1.3 \%)$, CABG surgery $(75 \%$ vs $66 \%$ ), and valve procedures ( $40 \%$ vs $39 \%$ ) (Table 2 ).

\section{Primary Outcomes}

The del Nido group had a greater proportion of patients who returned to spontaneous rhythm than the control group $(97.7 \%$ vs $81.6 \% ; P=.023)$, but this result did not reach statistical significance. Defibrillation after coronary reperfusion was similar between the del Nido and control groups $(4.7 \%$ vs $13.2 \% ; P=.244)$ and there was also no difference between the del Nido and control groups on the median number of defibrillation attempts required $(z=-1.31 ; P=.190)$. Fewer patients in the del Nido group required inotropic support compared with the control group $(65.1 \%$ vs $84.2 \% ; P=.050)$. Repeated measures analysis of all 4 time points found that for the del
Nido group, troponin levels after surgery did not increase as much as for the control group $(F=4.1 ; P=.040)$ (Figure 2), but this interaction term did not reach statistical significance. By 24 hours after admission to the CVICU, troponin levels reached $7.0 \pm 14.7$ in the control group whereas troponin levels were $2.3 \pm 2.1$ in the del Nido group $(P=.053)$.

\section{Secondary Outcomes}

There was no difference between the del Nido group and control group on CPB time (97 vs 103 minutes; $P=.288$ ), and although cross-clamp time was shorter for the del Nido group (70 vs 83 minutes; $P=.018$ ), the comparison did not reach statistical significance. After adjustment for preoperative ejection fraction measured by TEE, regression analysis found that the del Nido and control groups were similar on postoperative ejection fraction measured by TEE $(t=0.3 ; P=.787)$.

Incidence of STS-defined morbidity was low, with no strokes, perioperative myocardial infarctions, renal failure, or operative deaths in either group. The composite outcome of any STS-defined complication appeared lower for the del Nido group, although the analysis did not reach statistical significance ( $11.6 \%$ vs $26.3 \% ; P=.089)$. The proportion of patients with blood transfusions was similar for the del Nido and control groups ( $4 \%$ vs $7 \% ; P=.658)$, as was

TABLE 2. Patient characteristics by treatment group

\begin{tabular}{|c|c|c|c|}
\hline Characteristic & Control group $(n=41)$ & del Nido group $(n=48)$ & $P$ value \\
\hline Age (y) & $65.1 \pm 9.1$ & $65.3 \pm 7.9$ & .885 \\
\hline Female & $10(24)$ & $8(17)$ & .366 \\
\hline Body mass index & $30.6 \pm 5.0$ & $30.2 \pm 6.2$ & .779 \\
\hline Ejection fraction $(\%)$ & $57.6 \pm 11.4$ & $54.3 \pm 11.9$ & .187 \\
\hline Diabetes & $15(37)$ & $22(46)$ & .378 \\
\hline Hypertension & $32(78)$ & $35(73)$ & .576 \\
\hline Peripheral vascular disease & $4(10)$ & $2(4)$ & .408 \\
\hline Cerebrovascular disease & $5(12)$ & $6(13)$ & .965 \\
\hline Chronic pulmonary disease & $17(42)$ & $21(44)$ & .828 \\
\hline Dyslipidemia & $36(88)$ & $41(85)$ & .742 \\
\hline Preoperative creatinine & $1(0.8-1.2)$ & $1(0.8-1.2)$ & .794 \\
\hline Preoperative hematocrit $(\%)$ & $39.2 \pm 5.9$ & $39.3 \pm 4.8$ & .900 \\
\hline STS risk score $(\%)$ & $1.3 \pm 1.1$ & $1.4 \pm 1.0$ & .859 \\
\hline Elective status & $29(71)$ & $35(73)$ & .819 \\
\hline CABG surgery & $27(66)$ & $36(75)$ & .344 \\
\hline Isolated CABG surgery & $25(61)$ & $29(60)$ & .957 \\
\hline CABG/valve surgery & $2(5)$ & $7(15)$ & .170 \\
\hline Valve surgery & $16(39)$ & $19(40)$ & .957 \\
\hline Aortic valve & $15(37)$ & $14(29)$ & .457 \\
\hline Mitral valve & $1(2)$ & $5(10)$ & .212 \\
\hline
\end{tabular}

Values are presented as mean \pm standard deviation, median (interquartile range), or n (\%). STS, Society of Thoracic Surgeons; CABG, coronary artery bypass grafting. 


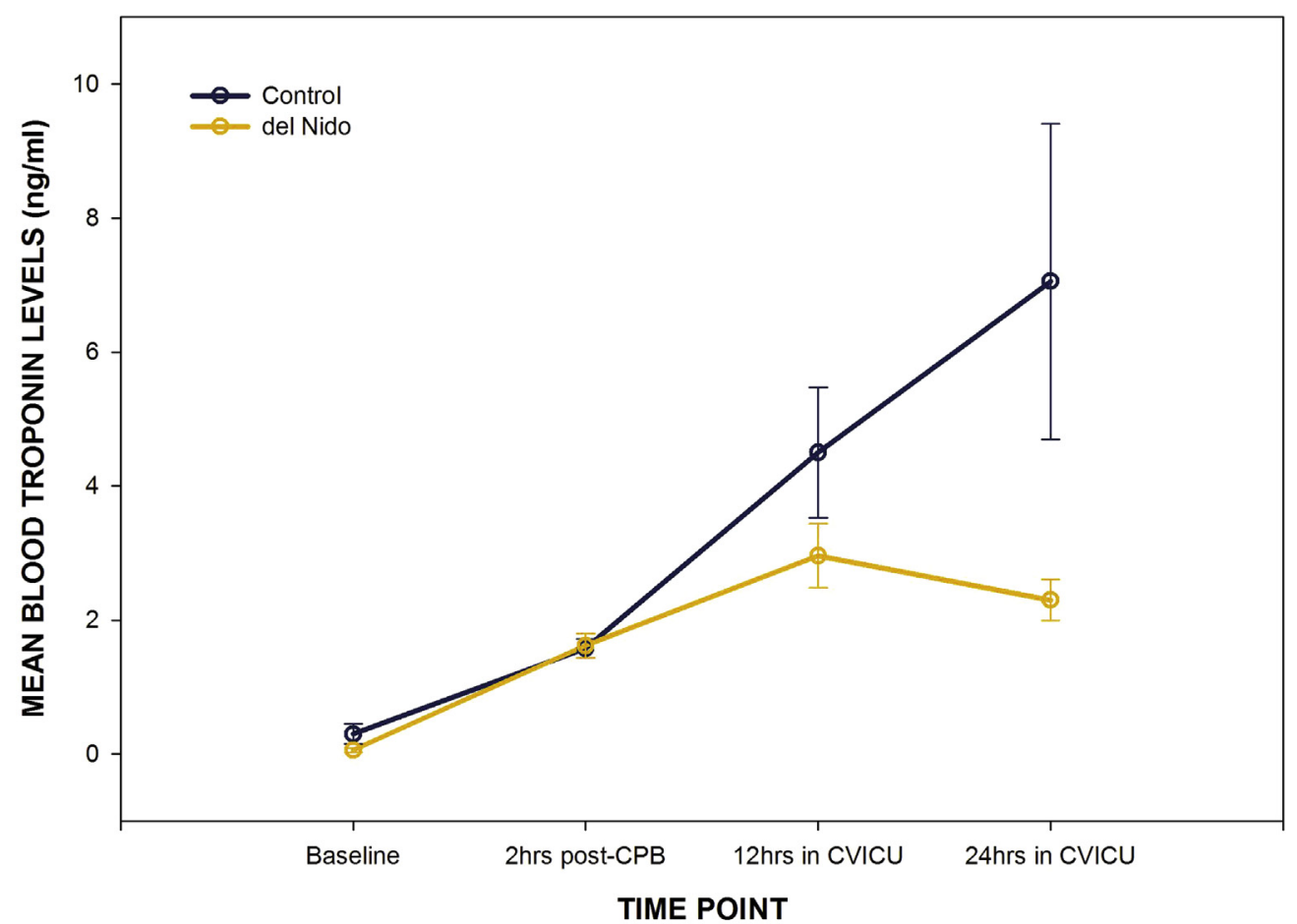

FIGURE 2. Mean \pm standard error troponin I levels in the 2 treatment groups from presurgery to 24 hours after cardiovascular intensive care unit ( $C V I C U$ ) admission. $C P B$, Cardiopulmonary bypass.

mean hematocrit at discharge $(31.6 \% \pm 4.9 \%$ vs $31.8 \% \pm 4.5 \% ; P=.882)$. None of the patients had a new $Q$ wave on electrocardiogram and only 1 control group patient showed ST-segment elevation.

The mean total volume of cardioplegia solution administered was significantly lower for the del Nido groups compared with the control group (1746.2 $\pm 852.5 \mathrm{~mL}$ vs $5077.8 \pm 2475.4 \mathrm{~mL} ; P<.001)$. The amount of crystalloid used in the mean volume of del Nido solution (1746.2 mL) was $1396.6 \mathrm{~mL}$. This amount was calculated using a 1:4 ratio of blood to crystalloid. The amount of crystalloid used in the mean volume of microplegia $(5077.8 \mathrm{~mL})$ was $114 \mathrm{~mL}$. This was calculated by adding the calculated amount of $44 \mathrm{~mL}$ potassium chloride, $40 \mathrm{~mL}$ magnesium sulfate, and $30 \mathrm{~mL}$ substrate-enriched monosodium aspartate/monosodium glutamate additive. Similarly, the median number of cardioplegia doses administered was also significantly lower for the del Nido group in comparison to the control group (1 [interquartile range, 1-2] vs 5 [interquartile range, 4-7]; $z=-7.39 ; P<.001)$. For the del Nido group, $28 \%$ required more than one dose of cardioplegia, whereas $100 \%$ of the control group required $>1$ dose $(P<.001)$.

\section{DISCUSSION}

The findings from this study indicate that the use of del Nido cardioplegia in both adult $\mathrm{CABG}$ and valve surgeries can be administered safely and with comparable levels of myocardial injury and inotropic support, and may yield significant benefits in surgical workflow in certain types of surgeries and surgical approaches. These findings are consistent with several previously reported observational studies. Although there are a multitude of cardioplegia formulas and techniques reported in the scientific literature, few studies compare the effects in a controlled prospective study design. Previous randomized controlled trials in myocardial protection have been limited to multidose and depolarizing cardioplegia solutions. A prospective, randomized study has yet to be performed on single-dose modified depolarizing strategies. The purpose of the present study was to compare the use of del Nido cardioplegia delivery to our current whole blood-based delivery protocol with the initial hypothesis that del Nido solution would be noninferior to our conventional strategy in both myocardial functional recovery and perioperative clinical outcome. Not only did our analyses support this hypothesis, but may also indicate some improvement in surgical workflow, such as shorter CPB and cross-clamp times and fewer doses of cardioplegia.

In a propensity score-matched review of consecutive patients presenting at New York Presbyterian Hospital for reoperative aortic valve replacement surgery, Sorabella and colleagues $^{18}$ found no significant differences in perioperative patient outcome between whole blood and del Nido cardioplegia protocols. The del Nido group received significantly less cardioplegia volume and the need for retrograde cardioplegia delivery. Researchers from the 
same institution analyzed 88 consecutive patients who presented with acute myocardial infarction for CABG surgery. Yerebaken and colleagues ${ }^{12}$ concluded no differences between the del Nido and whole blood cardioplegia groups in postoperative outcome. However, significant reductions in CPB duration and aortic cross-clamp time were realized in that high-risk cohort of patients. ${ }^{12}$ In another propensity score-matched review of 100 consecutive patients presenting for CABG surgery, Timek and colleagues ${ }^{13}$ compared outcome between del Nido cardioplegia and their conventional blood cardioplegia protocol. Despite the significant differences in total cardioplegia volume, number of doses, and route of delivery, del Nido cardioplegia delivered as a single antegrade dose provided equivalent myocardial protection in patients undergoing coronary revascularization.

The decision to use del Nido for patients with multivessel coronary artery disease has been a reported concern for programs, even those who routinely employ del Nido solution for other heart procedures. ${ }^{22}$ The maldistribution of cardioplegia delivery due to severe coronary disease may prevent penetration in ischemic areas of the myocardium. Strategies to provide uniform hypothermia of the heart may include retrograde cardioplegia, cardioplegia delivery down the saphenous vein grafts, and topical hypothermia. The use of retrograde cardioplegia at our institution has been considered a standard for the majority of patients presenting for CABG surgery. For retrograde administration, we elected to halve the dose of del Nido cardioplegia between routes to ensure proper distribution and arrest. Further, the use of topical ice lavage during valve procedures was also used at the discretion of the surgeon. Although we did not directly monitor myocardial temperature, we believe we adequately addressed our own concerns in ensuring uniform distribution and subsequent tissue warming.

Perioperative cardiac troponin levels did not increase as high in the del Nido group compared with the whole blood control group. At the 24-hour postoperative interval, troponin levels in the whole blood group failed to demonstrate a true peak value, whereas the trend in the del Nido group showed a decrease in biomarker production. According to the National Academy of Clinical Biochemistry Medicine Practice Guidelines, the time to first increase for cardiac troponin levels is approximately 3 to 4 hours, with peak levels occurring at approximately 18 hours after the onset of symptoms. ${ }^{23-25}$ Accordingly, we scheduled our biomarker assays to capture the initial ischemic insult, postoperative elevation and peak levels within the first 24 hours postsurgery. Other del Nido studies failed to show significant differences in blood troponin levels between study groups, but it may be related to the retrospective nature of the studies and the lack of standardization in the samples regarding time intervals and the number of tests. ${ }^{14,16}$ We believe the trend for a difference in peak troponin release may be multifactorial. First, the lower hematocrit in del Nido may have provided improved distribution throughout the microcirculation. As temperature decreases, the viscosity of whole blood increases. Our whole blood-based cardioplegia protocol may not have provided equal distribution of cardioplegia volume at hypothermic infusion temperatures. In vitro models have explained the profound influence that altering hematocrit and temperature change have on blood viscosity and fluid distribution. ${ }^{26}$ The resultant hematocrit delivery of del Nido was estimated between $6 \%$ and $7 \%$, whereas the hematocrit of whole blood cardioplegia was estimated to be delivered between $26 \%$ and $32 \%$. Lidocaine may also have a direct influence on tissue vasoactivity and microvascular response through action potential inhibition and increased nitric oxide release. ${ }^{27}$ Furthermore, we recognize the potential benefits that lidocaine may provide in preserving intracellular $\mathrm{pH}$ and limiting calcium ion influx. Our conventional whole blood technique did not include lidocaine as an additive; rather, a systemic bolus of $1 \%$ lidocaine was administered before removal of the aortic cross-clamp. The prolonged action of lidocaine when used in the cardioplegia formula may provide improved myocyte preservation during ischemic arrest. $^{7,28}$ Although the whole blood technique did incorporate magnesium sulfate as a natural calcium antagonist, the combined delivery of lidocaine and magnesium in del Nido may have provided further protection from injury. By inhibiting the influx of intracellular protons through sodium channel blockade and buffering agents such as carbonic anhydrase, glycogen stores inside the cells can be used to provide additional ATP stores during ischemia. Similar observations were reported by Gustavo and Voolaran. ${ }^{17,19}$ However, a retrospective analysis performed at Brigham and Women's Hospital ${ }^{29}$ reported that the presence of lidocaine in their modified del Nido formula was associated with a 4- to 5-fold increase in postoperative creatine kinase-MB values when compared with their standard whole blood cardioplegia. Despite these increases, operative and 1-year mortality rates were similar between groups. The authors concluded that lidocaine-containing cardioplegia can be administered safely for the first 60 minutes of aortic crossclamping. We believe a larger, controlled study of various lidocaine-enriched cardioplegia strategies is warranted. Future studies should include a more specific perioperative echocardiography protocol. More specifically, an intraoperative echocardiogram as a single assessment for myocardial function is likely not sufficient and additional tests at day 2 or 3 and 5 could provide better evidence. We also believe that future studies should include specific assessment for subendocardial function such as global longitudinal strain 24 hours before and 2 to 3 days after surgery. Global longitudinal strain is a very sensitive marker for subendocardial function and is well validated.

Mean aortic cross-clamp duration was shorter in the del Nido group. Although not statistically significant, this 
finding was attributed to the need for the surgeon to intermittently stop and deliver maintenance doses of cardioplegia in conventional depolarizing solutions. This finding was consistent with other studies comparing del Nido cardioplegia to conventional techniques, as well as other single-dose crystalloid strategies such as Custodiol (Essential Pharmaceuticals, LLC, Durham, NC). ${ }^{19,30}$ Although we realized a reduction in CPB duration, this difference also did not reach statistical significance. Continued expansion of del Nido into more complex patient and surgical profiles may further improve surgical workflow, including shorter CPB and cross-clamp times and fewer doses of cardioplegia.

\section{Limitations}

There were several limitations to our study. We compared the use of del Nido to our current whole blood cardioplegia protocol. Our findings may not be generalizable to the broader surgical community, because the multitude of variations in cardioplegia technique may not provide comparable results. Other del Nido comparative studies use more commonly reported Buckberg cardioplegia formulas as controls. In addition, the lack of surgical team blinding could introduce a source of bias to some of the outcome measures, although these variables were not captured by the surgical team and the surgical team did not have consensus regarding the preferred myocardial preservation method to be applied. The indications for inotropes were not standardized in this study and therefore may not represent an unbiased measure of myocardial protection. We also were unable to confirm a peak troponin value in the control group at 24 hours. Troponin values may have peaked even higher than what was reported in our analysis. We were unable to determine the long-term outcomes associated with elevated postoperative troponin values. Additionally, our eligibility criteria excluded high-risk cardiac surgery patients. Patients with previous cardiac surgery, requiring pharmacologic and mechanical cardiac support, and patients older than age 80 years were not considered for study. Further, the mean aortic cross-clamp times were 70 and 83 minutes for the del Nido and whole blood groups, respectively. As such, we were unable to substantiate the effects of del Nido for prolonged periods of arrest beyond 2 hours. Another limitation of this study is the small sample size, which influenced the power of some analyses. Given the interim analysis status for these results, a post hoc alpha spending function was applied and only comparisons that reached $P<.001$ were considered statistically significant. The use of this technique in combination with the small sample size resulted in not rejecting the null hypothesis for some analyses that met an unadjusted alpha level. In addition, although the results of the troponin analyses found a greater increase in values for the control group, the result did not reach statistical significance and observed power was $56 \%$ for this analysis, which requires replication in future studies for validation. However, we believe that these findings, in conjunction with previous retrospective literature, can serve as pilot data to support the undertaking of a larger randomized controlled trial, perhaps expanding the patient population and surgical procedures included.

\section{CONCLUSIONS}

Evidence from this study supports previous literature that suggests expanding the use of del Nido cardioplegia to routine adult cases may be safe and could improve streamline surgical workflow with comparable clinical outcomes. The difference in troponin levels should be investigated further because it may reflect myocardial protection associated with del Nido solution versus blood cardioplegia, especially in regard to vasodilation and microvascular response.

\section{Webcast}

You can watch a Webcast of this AATS meeting presentation by going to: https://aats.blob.core.windows.net/ media/17AM/2017-05-01/BallroomABC/05-01-17_ BallroomABC_1410_Fitzgerald.mp4.

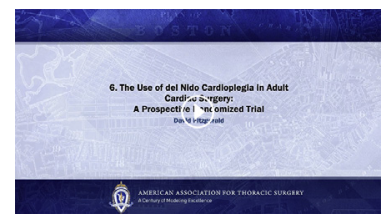

\section{Conflict of Interest Statement}

N.A. is a consultant for Medtronic, a member of the speaker's bureau for AtriCure, a proctor and member of the speaker's bureau for LivaNova, on the advisory board for Nido Surgical, and co-owner for Left Atrial Appendage Occlusion, LLC. All other authors have nothing to disclose with regard to commercial support.

Data were collected and maintained by Jane Lettmann, RN, CCRC; Rabia Ali, MS; Jill Bennick; Deborah Lamont, BSN, RN; and Graciela Pritchard, BS, at Inova Fairfax Hospital in Falls Church, Va. The perfusion team provided valuable support for this study as well. The authors thank their colleagues for their contributions and the cardiac surgical team at New York Presbyterian Hospital-Columbia for sharing their experiences and techniques.

\section{References}

1. Ovrum E, Tangen G, Tollofsrud S, Oystese R, Ringdal MA, Istad R. Cold blood
versus cold crystalloid cardioplegia: a prospective randomised study of 345
aortic valve patients. Eur J Cardiothorac Surg. 2010;38:745-9.
2. Reitz BA. Myocardial protection during cardiac surgery. Annu Rev Med. 1982;
33:151-62.
3. Melrose DG, Dreyer B, Bentall HH, Baker JBE. Elective cardiac arrest. Lancet.
1955;266:21-3.
4. Cordell AR. Milestones in the development of cardioplegia. Ann Thorac Surg.
1995;60:793-6. 
5. Fallouh HB, Chambers DJ. Is blood versus crystalloid cardioplegia relevant? Significantly improved protection may require new cardioplegic concepts. Interact CardioVasc Thorac Surg. 2008;7:1162-3

6. Sá M, Rueda FG, Ferraz PE, Chalegre ST, Vasconcelos FP, Lima RC. Is there any difference between blood and crystalloid cardioplegia for myocardial protection during cardiac surgery? A meta-analysis of 5576 patients from 36 randomized trials. Perfusion. 2012;27:535-46.

7. Matte GS, del Nido PJ. History and use of del Nido cardioplegia solution at Boston Children's Hospital. J Extra Corporeal Technol. 2012;44:98-103.

8. Govindapillai A, Hua R, Rose R, Friesen CH, O'Blenes SB. Protecting the aged heart during cardiac surgery: use of del Nido cardioplegia provides superior functional recovery in isolated hearts. J Thorac Cardiovasc Surg. 2013;146:940-8.

9. O'Blenes SB, Friesen CH, Ali A, Howlett S. Protecting the aged heart during cardiac surgery: the potential benefits of del Nido cardioplegia. J Thorac Cardiovasc Surg. 2011;141:762-70.

10. Harvey B, Shann KG, Fitzgerald D, Mejak B, Likosky DS, Puis L, et al. International pediatric perfusion practice: 2011 survey results. J Extra Corporeal Technol. 2012;44:186-93.

11. Kotani Y, Tweddell J, Gruber P, Pizarro C, Austin EH IIIrd, Woods RK, et al. Current cardioplegia practice in pediatric cardiac surgery: a North American multiinstitutional survey. Ann Thorac Surg. 2013;96:923-9.

12. Yerebakan H, Sorabella RA, Najjar M, Castillero E, Mongero L, Beck J, et al. Del Nido cardioplegia can be safely administered in high-risk coronary artery bypass grafting surgery after acute myocardial infarction: a propensity matched comparison. J Cardiothorac Surg. 2014;9:141.

13. Timek T, Willekes C, Hulme O, Himelhoch B, Nadeau D, Borgman A, et al. Propensity matched analysis of del Nido cardioplegia in adult coronary artery bypass grafting: initial experience with 100 consecutive patients. Ann Thorac Surgery. 2016;101:2237-41.

14. Smigla G, Jaquiss R, Walczak R, Bonadonna D, Kaemmer D, Schwimer C, et al Assessing the safety of del Nido cardioplegia solution in adult congenital cases. Perfusion. 2014;29:554-8.

15. Ota T, Yerebakan H, Neely RC, Mongero L, George I, Takayama H, et al. Shortterm outcomes in adult cardiac surgery in the use of del Nido cardioplegia solution. Perfusion. 2016:31:27-33.

16. Mick SL, Robich MP, Houghtaling PL, Gillinov AM, Soltesz EG, Johnston DR, et al. Del Nido versus Buckberg cardioplegia in adult isolated valve surgery. $J$ Thorac Cardiovasc Surg. 2015;149:626-36.

17. Guajardo Salinas GE, Nutt R, Rodriguez-Araujo G. Del Nido cardioplegia in low risk adults undergoing first time coronary artery bypass surgery. Perfusion. 2017; 32:68-73.
18. Sorabella RA, Akashi H, Yerebakan H, Najjar M, Mannan A, Williams MR, et al Myocardial protection using del Nido cardioplegia solution in adult reoperative aortic valve surgery. J Cardiac Surg. 2014;29:445-9.

19. Valooran GJ, Nair SK, Chandrasekharan K, Simon R, Dominic C. Del Nido cardioplegia in adult cardiac surgery - scopes and concerns. Perfusion. 2016;31:6-14.

20. Lan KKG, DeMets DL. Discrete sequential boundaries for clinical trials. Biometrika. 1983;70:659-63.

21. Reboussin DM, DeMets DL, Kim KM, Lan KKG. Computations for group sequential boundaries using the Lan-DeMets spending function method. Control Clin Trials. 2000;21:190-207.

22. Kim K, Ball C, Grady P, Mick S. Use of del Nido cardioplegia for adult cardiac surgery at The Cleveland Clinic: perfusion implications. J Extra Corporeal Technol. 2014:46:317-23.

23. Morrow DA, Cannon CP, Jesse RL, Newby LK, Ravkilde J, Storrow AB, et al. National Academy of Clinical Biochemistry Laboratory Medicine practice guidelines: clinical characteristics and utilization of biochemical markers in acute coronary syndromes. Circulation. 2007;115:e375.

24. Croal BL, Hillis GS, Gibson PH, Fazal MT, El-Shafei H, Gibson G, et al. Relationship between postoperative cardiac troponin I levels and outcome of cardiac surgery. Circulation. 2006;114:1468-75

25. Lasocki S, Provenchère S, Bénessiano J, Vicaut E, Lecharny J, Desmonts J, et al. Cardiac troponin I is an independent predictor of in-hospital death after adult cardiac surgery. Anesthesiology. 2002;97:405-11

26. Stammers AH, Vang SN, Mejak BL, Rauch ED. Quantification of the effect of altering hematocrit and temperature on blood viscosity. J Extra Corporeal Technol. 2003;35:143-51.

27. O'Brien JD, Howlett SE, Burton HJ, O'Blenes SB, Litz DS, Friesen CLH. Pediatric cardioplegia strategy results in enhanced calcium metabolism and lower serum troponin T. Ann Thorac Surg. 2009;87:1517-23.

28. Newton DJ, McLeod GA, Khan F, Belch JJ. Mechanisms influencing the vasoactive effects of lidocaine in human skin. Anaesthesia. 2007;62:146-50.

29. Yammine M, Neely RC, Loberman D, Rajab TK, Grewal A, McGurk S, et al. The use of lidocaine containing cardioplegia in surgery for adult acquired heart dis ease. J Cardiac Surg. 2015:30:677-84.

30. Hummel BW, Buss RW, DiGiorgi PL, Laviano BN, Yaeger NA, Lucas ML et al. Myocardial protection and financial considerations of Custodiol cardioplegia in minimally invasive and open valve surgery. Innovations (Phila) 2016;11:420-4

Key Words: cardiac surgery, cardioplegia, del Nido cardioplegia, RCT 